\title{
A pineapple flavor imitation by the note method
}

\author{
Guangyong $\mathrm{ZHU}^{1 *}$ (D), Genfa YU
}

\begin{abstract}
Pineapple is an aromatic tropical fruit and has attractive sweet odor. The aroma of pineapple is very popular and pineapple flavor has good prospects for application. Usually, flavor recipes are trade secrets. This paper deals with how to construct a pineapple flavor recipe and how to imitate a pineapple flavor with natural and synthetic aroma ingredients. By smelling and tasting pineapple fruit, the notes of pineapple flavor was identified as fruity note, winy note, vanilla-like note, vegetative note, beany note, sweet note and acidic note. In the light of the notes of pineapple flavor, the corresponding aromatic natural and synthetic ingredients having the same notes were choose to construct a pineapple flavor recipe. On the basis of the olfactory discrimination, the pineapple recipe was modified and adjusted for several times, and finally a desired pineapple flavor recipe was obtained. The blended flavor based on the recipe was harmonious and had typical natural odor characteristics of pineapple fruit.
\end{abstract}

Keywords: pineapple; flavor recipe; aroma; imitation; sensory analysis.

Practical Application: Pineapple flavor was imitated and a typical pineapple flavor recipe was obtained.

\section{Introduction}

Flavor is important in driving consumption (Murphy et al., 2008). Because characteristics of odor play a crucial role in the consumer preference and acceptance, flavors are very important in food industry (Zhu \& Xiao, 2015; Zhu et al., 2015). In general, flavors are mixtures of aroma constituents blended by synthetic and natural raw materials. The addition of flavoring adjuvants and agents in food is to give or help present an aroma or taste. Technology, art and their combination are embodiments of flavor science. The main 'tool' or 'device' is the flavorist's nose. Flavorists have high level of experiences in blending accords as well as in the perception of scents (Zhu \& Xiao, 2017). The exact molecules and ratios in flavor recipe are usually trade secrets (Epstein et al., 2015). From the viewpoint of a creative flavorist, the books about flavor creation are very few (Wright, 2011).

Usually, fruity aroma is a favorite scent for most of people. In the field of flavor formulation, the production of imitation fruity flavors is very important. Among many fruits, pineapple is an aromatic tropical fruit. Because of the attractive sweet odor of pineapple, it is widely used as processed juice, fresh fruit and canned fruit. In exotic foods, pineapple is often also used as an ingredient (Berger et al., 1985; Hui, 2010). Furthermore, pineapple flavor is widely used in food (Dias et al., 2012), beverage (Wu et al., 2005), ice-cream (Cai \& Chen, 1999), cigarette (Zhang et al., 2018), and so on. Thus, pineapple flavor imitation is of great significance. Note is defined as odor characteristic or a distinct flavor (Burdock, 2010; Fortineau, 2004). Pineapple flavor imitation by the note method was investigated in this work. The notes of pineapple flavor were identified and a typical pineapple flavor recipe was obtained. The compounding flavor based on the recipe has a characteristic odor of pineapple fruit, and is harmonious.

\section{Experimental methods}

\subsection{Materials}

All the natural and synthetic aroma ingredients for imitating pineapple flavor must be food grade. Robert Company provided the natural aroma products for blending pineapple flavor. The synthetic raw materials came from Shanghai Pu-Jie Fragrance Co., Ltd. These raw materials were used as received.

\subsection{Methods}

In the experiment, the note method (Zhu \& Xiao, 2015, 2017) was adopted to imitate pineapple flavor. The perception of flavor involves the combined human sense of smell and taste. By the human sense, numerous flavors can be recognized. Firstly, smelled and tasted pineapple fruit to identify odor characteristics and the notes of pineapple flavor as shown in Figure 1.

Secondly, smelled various synthetic and natural raw materials to be familiar with the odor characteristics of these raw material. When smelling the aroma ingredients, smelling slips was used to identify the odor characteristics of these ingredients. Dipped smelling slips into each of the raw materials and smelled the slips to identify the notes of these ingredients carefully as shown in Figure 2.

Thirdly, in the light of the notes of pineapple flavor identified, the corresponding aromatic natural and synthetic compounds which have the same notes were choose to construct an initial pineapple flavor recipe. On the basis of these notes of the raw materials and pineapple, ingredients were selected and their ratios were defined for imitation of pineapple flavor. Based on the pineapple flavor recipe designed initially, all the raw materials were weighed carefully in a bottle with a balance as shown in Figure 3. 


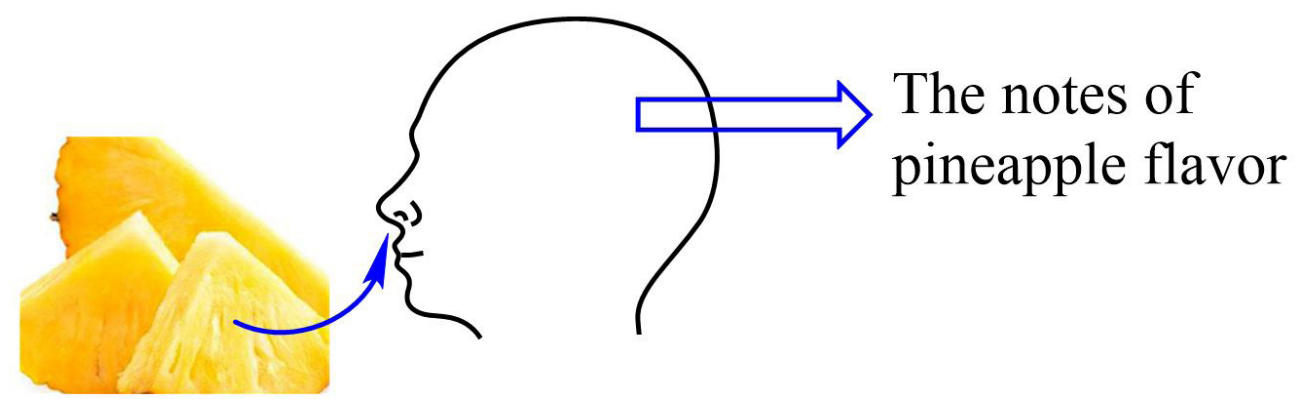

Figure 1. Identification of the notes of pineapple flavor.
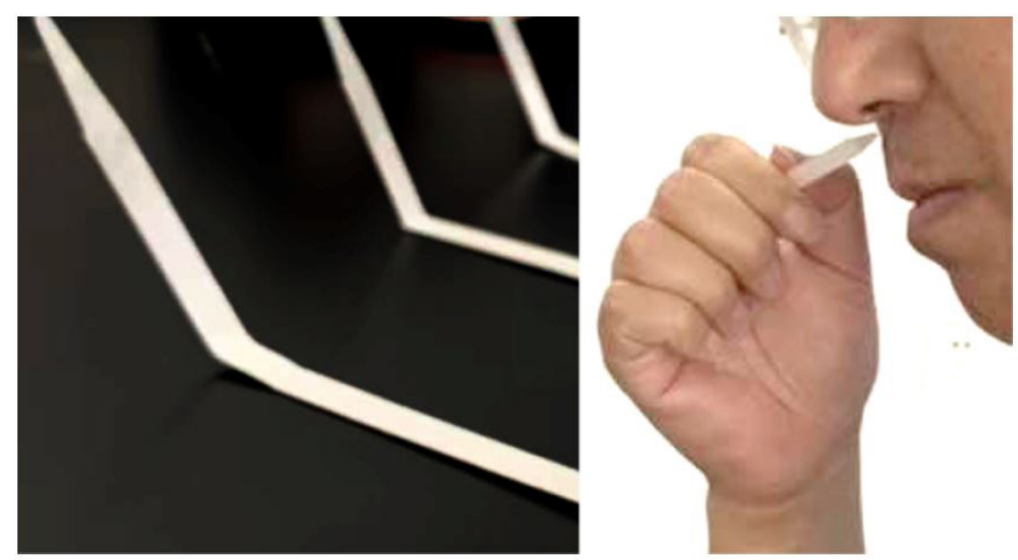

Figure 2. Identification of note of raw materials with smelling slips.

When all the ingredients were added completely in the bottle, shook the bottle to make sure that all the raw materials could be well mixed. Using smelling slip, the compounding pineapple flavor was evaluated. Based on the results of evaluation, the pineapple recipe was modified and adjusted several times. Finally, a desired pineapple flavor was imitated and its recipe was obtained.

\section{Results and discussion}

\subsection{The pineapple flavor notes identified by smelling and tasting}

In the light of the perception of pineapple fruit and literature (Zhou \& Xiao, 2007), the pineapple flavor notes identified are shown in Figure 4.

The pineapple flavor was divided into fruity, winy, vanilla-like, vegetative, beany, sweet and acidic note. Therefore, a pineapple flavor can be imitated by blending natural and synthetic raw materials with the corresponding notes. The notes of pineapple flavor play a crucial role in creating a pineapple flavor.

\subsection{Natural and synthetic ingredients selected for imitating a pineapple flavor}

Some aroma compounds were found in the volatiles of pineapple fruit. These compounds include esters, aldehydes, alcohols, acids, lactones and so on (Steingass et al., 2015). These aroma compounds can be used in the pineapple flavor formula. Of course, although some aroma ingredients were not found in pineapple fruit, these raw materials can also be adopted for

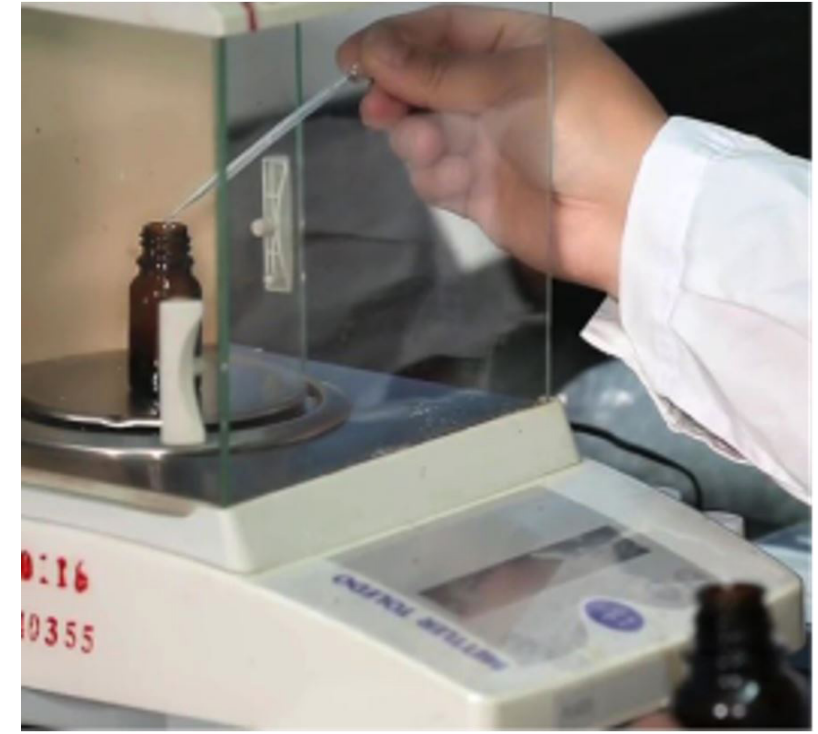

Figure 3. The process of blending pineapple flavor.

imitating pineapple flavor. The selection of raw materials is mainly based on the notes of these ingredients.

In the light of the notes of pineapple flavor and the notes of aroma raw materials, various raw materials were selected to imitate pineapple flavor. The fruity note is the main note in a pineapple flavor. It is very important for the imitation of pineapple flavor. Aliphatic esters usually have fruity odor. They 


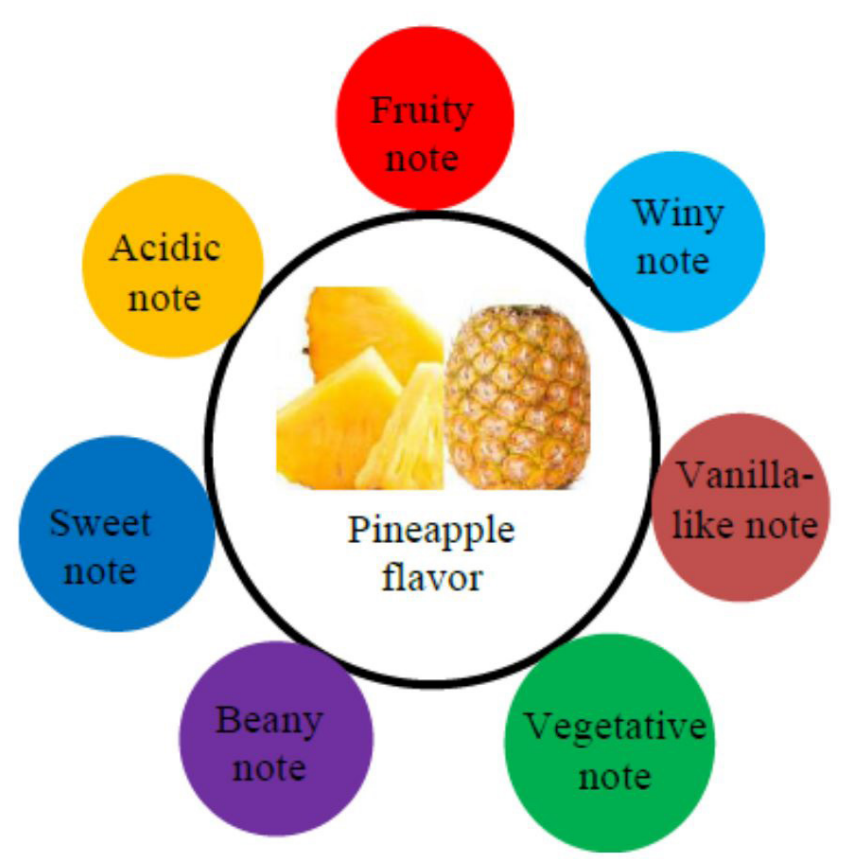

Figure 4. The notes of pineapple flavor identified by smelling and tasting.

contribute to almost all fruity aroma. Some aliphatic esters have a particular aroma of fruit. However, most of them have a nonspecific fruity odor (Morrill et al., 2017; Surburg \& Panten, 2006). Aliphatic esters are usually adopted to provided fruity odor in flavor creation. The aliphatic esters selected for imparting fruity note were as follows: allyl hexanoate, allyl heptanoate, allyl cyclohexanepropionate, ethyl 3-methylthiopropionate, methyl 3-methylthiopropionate, ethyl butyrate, isoamyl acetate, isoamyl butyrate and ethyl acetate. Allyl hexanoate has pineapple-like, fruity aroma and taste. Taste characteristics of allyl heptanoate are also pineapple-like, fruity when its concentration is $10 \mathrm{ppm}$. Allyl cyclohexanepropionate is colorless liquid with pineapple aroma. Allyl cyclohexanepropionate has pineapple fruity taste characteristics when its concentration is $30 \mathrm{ppm}$. Allyl hexanoate, allyl heptanoate and allyl cyclohexanepropionate are the main ingredients to impart odor characteristics of pineapple. The odor characteristics of ethyl 3-methylthiopropionate are pineapple-like and citrus-like. At low concentration, methyl-3-methylthiopropionate has a sweet pineapple aroma. The addition of small quantities of methyl 3-methylthiopropionate and ethyl 3-methylthiopropionate can improve natural pineapple characteristics. The odor characteristics of ethyl butyrate is fruity, somewhat pineapple-like. A typical banana aroma is the odor characteristics of isoamyl acetate (Morrill et al., 2017; Logan \& Rumbaugh, 2012). The odor characteristics of isoamyl butyrate is fruity, reminiscent of banana, apricot and pineapple. Ethereal fruity odor is the characteristics of ethyl acetate, which also smells like brandy and pineapple (Burdock, 2010; Surburg \& Panten, 2006; Bromfield-Lee \& Oliver-Hoyo, 2009). These three esters can enrich the fruity note of pineapple.

Except for esters, some lactones also have fruity note, and take on various fruity odor. A fruity, peach-like aroma is the odor characteristics of $\gamma$-undecalactone. $\gamma$-Nonalactone takes on coconut aroma (Burdock, 2010; Surburg \& Panten, 2006; Zhu et al., 2013). The two raw materials were selected to enrich the fruity note of pineapple.

Citral smells like lemon fruit and has been widely used in citrus flavors (Burdock, 2010). In pineapple flavor, citral was selected to enrich the fruity note of pineapple.

Sweet orange oil is a natural raw material derived from sweet orange peel. It has fresh, sweet, fruity odor and smells like orange fruit. Lemon oil, which smells like fresh lemon fruit, is also a natural raw material derived from lemon peel. The two oils were selected in pineapple flavor. On the one hand, the natural feeling of pineapple flavor can be enhanced by addition of the two natural raw materials. On the other hand, natural products can suppress the harshness of synthetics, round off the note and produce homogeneity (Burdock, 2010; Surburg \& Panten, 2006).

Because some lactones also contribute to beany note, the ingredients to impart beany note in pineapple flavor selected were as follows: $\gamma$-valerolactone, $\gamma$-hexalactone, and $\gamma$-heptalactone. All the three lactones have sweet odor with beany characteristics (Burdock, 2010; Surburg \& Panten, 2006).

The odor of acetic acid is pungent and strong. Acetic acid has a typical vinegar aroma, and has been widely used in fruit flavors. Acetic acid was selected to impart acidic note in pineapple flavor. In pineapple flavor, acetic acid can also be used to accentuate fruity notes (Burdock, 2010; Zhu et al., 2013).

Maltol and furaneol were two raw materials selected for imparting sweet note. Maltol takes on sweet, caramel-butterscotch odor. As a taste intensifier, maltol can provide sweet note and caramel note in pineapple flavor (Burdock, 2010). With a burnt pineapple odor, furaneol can also give sweet note in flavor formula (Zhu et al., 2015).

Vanillin takes on a characteristic, vanilla-like aroma. Its taste is very sweet. Therefore, vanillin was selected for imparting vanilla-like odor in pineapple flavor (Burdock, 2010; Surburg \& Panten, 2006).

Ethyl hexanoate and ethyl heptanoate were selected to give the winy note in pineapple flavor. Ethyl hexanoate has a winy and fruity odor. It also has a pineapple-banana note. Ethyl heptanoate takes on a winy-brandy and fruity odor (Burdock, 2010; Surburg \& Panten, 2006).

Methional was selected to impart vegetative note in pineapple flavor. It takes on a strong onion odor, and has taste characteristics of potato, tomato, seafood and vegetative nuances at low concentration (Burdock, 2010).

Propylene glycol and ethanol are common solvents used in flavor. In pineapple flavor, the two materials were selected as solvents.

\subsection{A typical pineapple flavor recipe obtained by the note method}

For imitation of pineapple flavor, the ratios of the selected raw materials were defined and an initial pineapple flavor recipe was designed. Based on the initial pineapple flavor recipe, a 
Table 1. A typical pineapple flavor recipe obtained by the note method.

\begin{tabular}{|c|c|c|}
\hline No & Flavor ingredient & wt $\%$ \\
\hline 1 & Allyl hexanoate & 10.83 \\
\hline 2 & Allyl heptanoate & 10.69 \\
\hline 3 & Allyl cyclohexanepropionate & 4.72 \\
\hline 4 & 1\% Ethyl 3-methylthiopropionate & 0.15 \\
\hline 5 & $1 \%$ Methyl 3-methylthiopropionate & 0.17 \\
\hline 6 & Ethyl butyrate & 0.29 \\
\hline 7 & Isoamyl acetate & 0.31 \\
\hline 8 & Isoamyl butyrate & 0.79 \\
\hline 9 & Ethyl acetate & 0.14 \\
\hline 10 & Sweet orange oil & 8.70 \\
\hline 11 & Lemon oil & 0.75 \\
\hline 12 & Citral & 0.02 \\
\hline 13 & $\gamma$-undecalactone & 0.63 \\
\hline 14 & $\gamma$-nonalactone & 0.64 \\
\hline 15 & Vanillin & 1.57 \\
\hline 16 & Maltol & 1.82 \\
\hline 17 & $10 \%$ furaneol & 0.26 \\
\hline 18 & Ethyl hexanoate & 0.13 \\
\hline 19 & Ethyl heptanoate & 0.67 \\
\hline 20 & $\gamma$-valerolactone & 0.23 \\
\hline 21 & $\gamma$-hexalactone & 0.21 \\
\hline 22 & $\gamma$-heptalactone & 0.01 \\
\hline 23 & Acetic acid & 0.01 \\
\hline 24 & 1\% Methional & 0.01 \\
\hline 25 & Pineapple base & 2.50 \\
\hline 26 & Ethanol & 40.7 \\
\hline 27 & Propylene glycol & 13.05 \\
\hline 28 & Total & 100 \\
\hline
\end{tabular}

pineapple flavor was blended and the odor characteristics were evaluated by nose with smelling slips. In the light of the odor evaluation by smelling, the pineapple flavor recipe was modified and adjusted for several times and finally a desired pineapple flavor was obtained. Table 1 shows a typical pineapple flavor recipe.

The pineapple flavor blended in the light of Table 1 has a harmonious and fresh pineapple aroma. The characteristics odor of natural pineapple fruit is obvious. In the experiment, a pineapple flavor was successfully imitated using the note method.

\section{Conclusions}

The paper deals with development of a pineapple flavor. The pineapple flavor was imitated successfully by the note method. By the perception of pineapple fruit, the pineapple flavor notes were identified. Fruity note, winy note, vanilla-like note, vegetative note, beany note, sweet note and acidic note constitute pineapple flavor. In the light of the notes of pineapple flavor, the corresponding aromatic natural and synthetic ingredients having the same notes were selected, and a pineapple flavor recipe was constructed. The pineapple recipe was modified and adjusted for several times based on the olfactory discrimination, and finally a desired pineapple flavor recipe was obtained. The pineapple flavor compounded on the biases of the recipe was harmonious and had typical natural odor characteristics of pineapple fruit.

\section{Acknowledgements}

The authors thank Shanghai Alliance Program (LM201844) for Financial support.

\section{References}

Berger, R. G., Drawert, F., Kollmannsberger, H., Nitz, S., \& Schraufstetter, B. (1985). Volatiles in pineapple fruit and their sensory properties. Journal of Agricultural and Food Chemistry, 32(2), 232-235. http:// dx.doi.org/10.1021/jf00062a019.

Bromfield-Lee, D. C., \& Oliver-Hoyo, M. T. (2009). An esterification kinetics experiment that relies on the sense of smell. Journal of Chemical Education, 86(1), 82-84. http://dx.doi.org/10.1021/ed086p82.

Burdock, G. A. (2010). Fenaroli's handbook of flavor ingredients. Boca Raton: CRC Press.

Cai, Y., \& Chen, Y. (1999). Flavor selection in ice-cream production. Food Industries, 1, 2-3.

Dias, N. A. A., Lara, S. B., Miranda, L. S., Pires, I. S. C., Pires, C. V., \& Halboth, N. V. (2012). Influence of color on acceptance and identification of flavor of food by adults. Food Science and Technology (Campinas), 32, 296-302.

Epstein, J. L., Castaldi, M., Patel, G., Telidecki, P., \& Karakkatt, K. (2015). Using flavor chemistry to design and synthesize artificial scents and flavors. Journal of Chemical Education, 92(5), 954-957. http://dx.doi.org/10.1021/ed500615a.

Fortineau, A.-D. (2004). Chemistry perfumes your daily life. Journal of Chemical Education, 81(1), 45-50. http://dx.doi.org/10.1021/ed081p45.

Hui, Y. H. (2010). Handbook of fruit and vegetable flavors. New Jersey: John Wiley and Sons, Inc. http://dx.doi.org/10.1002/9780470622834.

Logan, J. L., \& Rumbaugh, G. E. (2012). The chemistry of perfume: a laboratory course for nonscience majors. Journal of Chemical Education, 89(5), 613-619. http://dx.doi.org/10.1021/ed2004033.

Morrill, L. A., Kammeyer, J. K., \& Garg, N. K. (2017). Spectroscopy 101: A practical introduction to spectroscopy and analysis for undergraduate organic chemistry laboratories. Journal of Chemical Education, 94(10), 1584-1586. http://dx.doi.org/10.1021/acs.jchemed.7b00263.

Murphy, M. M., Douglass, J. S., Johnson, R. K., \& Spence, L. A. (2008). Drinking flavored or plain milk is positively associated with nutrient intake and is not associated with adverse effects on weight status in US children and adolescents. Journal of the American Dietetic Association, 108(4), 631-639. http://dx.doi.org/10.1016/j.jada.2008.01.004. PMid:18375219.

Steingass, C. B., Jutzi, M., Müller, J., Carle, R., \& Schmarr, H.-G. (2015). Ripening-dependent metabolic changes in the volatiles of pineapple (Ananas comosus (L.) Merr.) fruit: II. Multivariate statistical profiling of pineapple aroma compounds based on comprehensive twodimensional gas chromatography-mass spectrometry. Analytical and Bioanalytical Chemistry, 407(9), 2609-2624. http://dx.doi. org/10.1007/s00216-015-8475-y. PMid:25651901.

Surburg, H., \& Panten, J. (2006). Common fragrance and flavor materials. Weinheim: WILEY-VCH Verlag GmbH \& Co. http:// dx.doi.org/10.1002/3527608214.

Wright, J. (2011). Flavor creation. Carol Stream: Allured Business Media.

Wu, G.-X., Zhang, C.-f., \& Li, Y. (2005). Study on the suspension beverage of honeyed shaddock grain. Food Science and Technology (Campinas), 5, 42-44.

Zhang, S., Li, B., Zhou, S., Liu, S., Wang, M., \& Liu, Z. (2018). Application of design-expert in study on optimizing formulation of fruit-flavored e-cigarette liquid. Light Industry Science and Technology, 34, 25-27.

Zhou, Y., \& Xiao, Z. (2007). Preparation technology of flavors. Beijing: China Textile and Apparel Press. 
Zhu, G., \& Xiao, Z. (2015). Study on creation of an indocalamus leaf flavor. Food Science and Technology (Campinas), 35(4), 647-651. http://dx.doi.org/10.1590/1678-457X.6770.

Zhu, G., \& Xiao, Z. (2017). Creation and imitation of a milk flavour. Food \& Function, 8(3), 1080-1084. http://dx.doi.org/10.1039/ C7FO00034K. PMid:28154863.
Zhu, G., Xiao, Z., Zhou, R., \& Lei, D. (2015). Preparation and simulation of a taro flavor. Chinese Journal of Chemical Engineering, 23(10), 1733-1735. http://dx.doi.org/10.1016/j.cjche.2015.07.026.

Zhu, G., Xiao, Z., Zhou, R., Zhu, Y., \& Niu, Y. (2013). Study on development of a fresh peach flavor. Advanced Materials Research, 781-784, 15701573. http://dx.doi.org/10.4028/www.scientific.net/AMR.781-784.1570. 